\title{
Living with microbes and stars: the discontinuous universe of natural organic matter
}

\author{
Hertkorn N. ${ }^{1}$, Harir M. ${ }^{1}$, Gonsior M. ${ }^{2}$, Schmitt-Kopplin Ph. ${ }^{1}$ \\ ${ }^{1}$ Helmholtz-Center Munich - HMGU, German Research Center for Environmental Health, Research \\ Unit Analytical Biogeochemistry (BGC), 85758 Neuherberg, Germany; hertkorn@helmholtz- \\ muenchen.de \\ ${ }^{2}$ University of Maryland Center for Environmental Science, Chesapeake Biological Laboratory, \\ Solomons, USA
}

Keywords: NOM, spectroscopy, chemical evolution, ecosystem, element cycles

doi: 10.36291/HIT.2019.hertkorn

Various astrophysical and astrochemical environments and conditions readily generate mixtures of complex molecules, commonly in the temperature range from $2500-$ $2.725 \mathrm{~K}$ (the temperature of the cosmic microwave background). The compositional diversity of these molecules exceeds that of our current terrestrial biomolecules by a substantial margin. In the solar system, carbonaceous chondrites which show carbon contents up to $5 \%$ are remnants from the initial times of solar system formation 4.54 billion years (Ga) ago; very few other geological traces from the Hadean epoch ( $>4 \mathrm{Ga}$ ) remain on Earth. Recently discovered organomagnesium compounds in meteorites (CHOMg) are indicators of thermal and shock stress during meteorite formation, i.e. they are the first identified geomolecules to relate organic molecular diversity and conditions of meteorite formation. CHOMg compounds in meteorites can be seen as conceptual soil analogues which existed at the time of the formation of the earth and even several millions of years before that. They connect the previously known set of extraterrestrial abiotic CHNOS molecules and the mineral phase. Thermostable CHOMg compounds might have selectively preserved specific organic molecules through organo-mineral-interaction over geologic time scales and are therefore relevant in origin of life studies.

Current terrestrial extreme environments such as Yellowstone National Park are organic chemo-diversity hot spots and reveal an unique chemo-diversity that has not yet been observed anywhere in aquatic surface environments on Earth. The formation of NOM preceded the evolution of life and at any time in Earth's history, coevolution occurred between prebiotic/abiotic molecules, NOM and primitive and higher forms of life. The near continuum of binding sites available to ions and organic molecules acts to buffer against environmental and chemical extremes in the geo- and biosphere, which could damage life because of their potent reactivity. This key supportive role of NOM in life processes is sustained by strong interactions between biological and geochemical cycles.

Modern organic structural spectroscopy enables a thorough description of NOM molecular features which reflect ecosystem conditions and formation history. Hence, NOM from terrestrial (soil), freshwater (lakes, rivers, groundwater), marine (estuarine, surface and deep ocean), atmospheric (secondary organic aerosols) and extraterrestrial sources show distinct individual characteristics which in conjunction will enable a lively representation of our boundless carbon and all other element cycles. This contribution will use recent examples of molecular characterization of NOM from various sources to illustrate avenues to a holistic contemplation of our Earth in an evolving universe. 